\title{
Effects of Endophytic Infection by Fusarium moniliforme on Corn Growth and Cellular Morphology
}

\author{
I. E. Yates, C. W. Bacon, and D. M. Hinton, Toxicology and Mycotoxin Research Unit, Richard B. Russell Agri- \\ cultural Research Center, USDA/ARS, P.O. Box 5677, Athens, GA 30604
}

\begin{abstract}
Yates, I. E., Bacon, C. W., and Hinton, D. M. 1997. Effects of endophytic infection by Fusarium moniliforme on corn growth and cellular morphology. Plant Dis. 81:723-728.
\end{abstract}

Kernels of corn, Zea mays, were inoculated with Fusarium moniliforme to analyze seedling growth and development during endophytic, symptomless infection. In planta $F$. moniliforme distribution and seedling growth, expressed as shoot diameter, plant height, leaf length, and dry weight, were examined weekly for 28 days after planting. Even though no visible disease symptoms developed, $F$. moniliforme was isolated from most segments taken from seedlings grown from inoculated, but not noninoculated, kernels from the earliest to the latest sampling. $F$. moniliforme did not alter the rate or percentage of kernel germination, but seedlings grown from inoculated kernels had suppressed shoot diameter, plant height, leaf length, and plant weight 7 days after planting. However, seedling growth from inoculated kernels was similar to or greater than that from noninoculated kernels at 28 days. Histological modifications in seedlings grown from inoculated kernels included accelerated lignin deposition in shoots and modified chloroplast orientation in leaves. In summary, gross morphology and histology were altered in corn seedlings during symptomless, endophytic infection by $F$. moniliforme.

Additional keywords: anatomy, roots

Fusarium moniliforme J. Sheld. (teleomorph Gibberella fujikuroi (Sawada) Ito in Ito \& K. Kimura) has a wide host range, including major food crops such as corn (Zea mays L.) $(5,7,18)$. The vegetative and reproductive tissues of a developing corn plant become infected, and up to $90 \%$ of some seed lots have been infected with $F$. moniliforme $(18,20,32)$. The response of corn to $F$. moniliforme infection depends on the fungal strain. Some fungal isolates have been associated with severe disease symptoms, including blight of seedlings and rot of kernels, roots, and stalks $(18,19)$. In contrast, other strains infect both reproductive and vegetative structures in symptomless colonization of the corn plant ( 1 , 16,20).

Evidence from field and laboratory studies support the hypothesis that $F$. moniliforme strains that cause symptomless infection of corn plants are endophytes $(2,20)$. $F$. moniliforme has been documented by several investigators as common in both diseased and symptomless plant parts (12, $15,19,20)$. In addition, microscopic studies

Corresponding author: I. E. Yates

E-mail: iyates@asrr.arsusda.gov

Accepted for publication 25 March 1997.

Publication no. D-1997-0509-04R

This article is in the public domain and not copyrightable. It may be freely reprinted with customary crediting of the source. The American Phytopathological Society, 1997. of colonization of corn plants have demonstrated that hyphae are restricted to intercellular spaces during symptomless infection, compared to hyphae of disease-causing strains, which are found in both intercellular and intracellular sites (2,24). Corn plants with endophytic, symptomless infections by $F$. moniliforme create a dilemma for evaluating crop production advantages and disadvantages for this association. Other fungal endophytes benefit the growth of their plant hosts $(9,26)$. Most $F$. moniliforme strains, however, produce toxins associated with putative harmful effects on animal and human health (21-23).

Even though the deleterious effects on corn plant growth and development have been documented for disease-causing F. moniliforme strains $(18,32)$, the response of corn plants to symptomless, endophytic infection has not been analyzed. The objective of this study was to investigate both the in planta distribution and the morphological and cellular development of corn seedlings grown under controlled environmental conditions after inoculation of kernels with an endophytic strain of $F$. moniliforme that causes symptomless infection.

\section{MATERIALS AND METHODS}

Preparation of fungal inoculum. A strain of F. moniliforme, designated RRC 374 (mating population $\mathrm{A}$, + mating type), was isolated from symptomless, infected corn kernels after surface-disinfection (4). Spores were stored at $5^{\circ} \mathrm{C}$ on silica gel (25) until inoculated on potato dextrose agar (PDA) to initiate cultures for experimentation. Cultures were maintained at 25 to $27^{\circ} \mathrm{C}$ with a $12 \mathrm{~h}$ light/dark cycle for 15 to 21 days. Inoculum consisting of both conidia and mycelia was obtained by flooding the agar surface of a culture with $10 \mathrm{ml}$ of sterile distilled water. The inoculum concentration averaged $10^{6}$ to $10^{9} \mathrm{CFU} / \mathrm{ml}$, a range previously demonstrated to be satisfactory for infection (4).

Inoculation of kernels and growth of corn. Kernels of commercial sweet corn, cv. Silver Queen, were used as the source of plants. Both surface- and internal-borne fungi of kernels were reduced by modifying procedures developed by Daniels (10). Sterile vessels and sterile distilled water were used. Specifically, kernels placed in plastic cups were covered with $5.25 \%$ sodium hypochlorite, agitated vigorously for 10 min, rinsed twice with water, imbibed in fresh water for $4 \mathrm{~h}$ at $25^{\circ} \mathrm{C}$, heat-treated for $5 \mathrm{~min}$ at $60^{\circ} \mathrm{C}$, and rinsed in cool water. Noninoculated control kernels were placed directly in petri dishes containing $5 \mathrm{ml}$ of sterile distilled water. The remaining kernels were submerged in fungal inoculum for $3 \mathrm{~min}$ at room temperature before being placed in dishes containing $5 \mathrm{ml}$ of sterile distilled water. Noninoculated and inoculated kernels were incubated at $25^{\circ} \mathrm{C}$ for $12 \mathrm{~h}$.

Kernels with enlarged embryo axes were selected for planting in growth medium consisting of moistened kitty litter (Special Kitty, unscented, Wal-Mart Stores, Inc., Bentonville, AR) contained in $10.2-\mathrm{cm}^{3} \mathrm{com}-$ partments of plastic flats (T. O. Plastics, Inc., Minneapolis). Planting depth was standardized by placing each kernel in a 1.5$\mathrm{cm}$ deep depression in the growth medium prior to covering the kernel. Flats received $300 \mathrm{ml}$ of water from the bottom of trays daily during the first week. Thereafter, each seedling was fertilized daily with $30 \mathrm{ml}$ of water containing 0.12 g of Peters 20-20-20 (N-P-K, Grace-Sierra Horticultural Products Co., Milpitas, CA). Seedlings were grown in a growth chamber at 29 to $32^{\circ} \mathrm{C}$ with $16 \mathrm{~h}$ of light at an intensity of 256 . $\mathrm{m}^{-2} \cdot \mathrm{s}^{-1}$ and at 21 to $26^{\circ} \mathrm{C}$ for $8 \mathrm{~h}$ of darkness.

A total of 192 plants was analyzed (2 treatments $\times 4$ sampling dates $\times 8$ plants per sampling date $\times 3$ repeated experiments). Plant number and experiment duration were optimized to avoid overcrowding plants in the growth chamber. Seed germination, judged as coleoptile emergence, was monitored daily for 6 days after plant- 
ing. Shoot diameter (millimeters), plant height (centimeters) at the juncture with the growth medium, and length of the longest leaf (centimeters) were measured weekly for 4 weeks. Half of the plants harvested each week were analyzed for dry weight, and the other half were used for fungal isolation and microscopic studies. Samples were weighed after drying at $65^{\circ} \mathrm{C}$ for 7 days. The experimental design was a randomized complete block (14).

Data analysis. Seed germination was expressed as the percentage of kernels with emerging coleoptiles on a daily and cumulative basis. Cumulative coleoptile emergence was treated by automated curve-fitting analyses (TableCurve, Jandel Scientific, San Rafael, CA). Data were compared for significant differences, based on the $t$ test (14) $(P \leq 0.05)$, between inoculated and noninoculated kernels at each sampling date for daily and cumulative germination and for seedling shoot diameter, plant height, leaf length, and plant weight, as well as for root weight at 28 days after planting.

Fungal distribution and microscopy. Plant parts were surface-disinfected by washing in full-strength commercial bleach $(5.25 \% \mathrm{NaOCl})$ for $10 \mathrm{~min}$ and rinsing twice in sterile distilled water. In planta distribution of $F$. moniliforme was determined by placing 0.5 - to $1.0-\mathrm{mm}$ surfacedisinfected segments from the root, mesocotyl, node, stem, and oldest leaf on PDA. A total of 10 segments per plant part from each of 48 plants was analyzed during the experiments. Segments from which F. moniliforme grew on PDA were scored as positive, and the percentage of positive responses was calculated for each plant part.

Observed differences in tissue rigidity at the shoot-mesocotyl junction between seedlings grown from $F$. moniliforme-inoculated and noninoculated kernels prompted

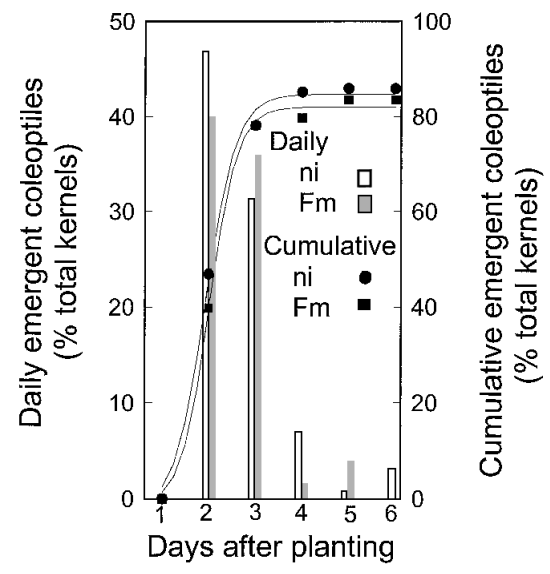

Fig. 1. Germination of noninoculated (ni) and Fusarium moniliforme-inoculated (Fm) corn kernels based on daily (bars, $P \geq 0.05$ ) and cumulative (lines) coleoptile emergence. For cumulative germination, the symbols represent the means and lines represent the derived sigmoid curves; $R^{2}=0.97, \mathrm{SE}=5.3 \%$. analysis of lignification in over 100 freehand sections of fresh stem tissue. Sections were placed on microscope slides and examined immediately in a drop of a saturated solution of phloroglucinol (ICN Pharmaceutical, Inc., Cleveland) in $18 \%$ HCL, a procedure that causes lignin to stain pink (27).

Fixed specimens from a weekly minimum of 24 seedlings grown from noninoculated and F. moniliforme-inoculated kernels were examined for plant anatomical features and fungal distribution. Plant materials consisted of (i) the root at $2 \mathrm{~mm}$ from the kernel; (ii) the shoot at the mesocotyl junction; and (iii) the oldest leaf at the midpoint of the length. Samples for scanning electron microscopy (SEM) were fixed overnight at $4^{\circ} \mathrm{C}$ in $4 \%$ glutaraldehyde in $0.1 \mathrm{M}$ sodium cacodylate buffer, $\mathrm{pH} 7.2$, and dehydrated in an ethanol series of $70,80,90$, and $100 \%$ for 30 min each. Samples were critical-point dried with $\mathrm{CO}_{2}$, mounted on colloidal silver on aluminum stubs, coated with gold palladium in a sputter coater, and examined at $5 \mathrm{kV}$ with a scanning electron microscope (JSM-6400V, JEOL, USA, Inc., Peabody, MA). Specimens for light microscopy (LM) and transmission electron microscopy (TEM) were prefixed overnight, as for SEM, rinsed in $0.1 \mathrm{M}$ sodium cacodylate buffer three times for $15 \mathrm{~min}$ each, postfixed for $1 \mathrm{~h}$ in an ice bath with $1 \% \mathrm{OsO}_{4}$ in $0.1 \mathrm{M}$ sodium cacodylate buffer, rinsed in distilled water three times for $15 \mathrm{~min}$ each, and dehydrated in an ethanol series of 50, 70, 90, and $100 \%$ for $30 \mathrm{~min}$ each. Specimens were infiltrated with a series of $100 \%$ ethanol/ Spurr's resin (28) of 50:50, 30:70, and 0:100 for $1 \mathrm{~h}$ each and embedded in resin at $60^{\circ} \mathrm{C}$ for $48 \mathrm{~h}$. Thick sections $(0.5 \mu \mathrm{m})$ were cut with an ultramicrotome (Ultracut E, Reichert-Jung, Vienna), transferred to glass slides, stained for 1 to 2 min with $1 \%$

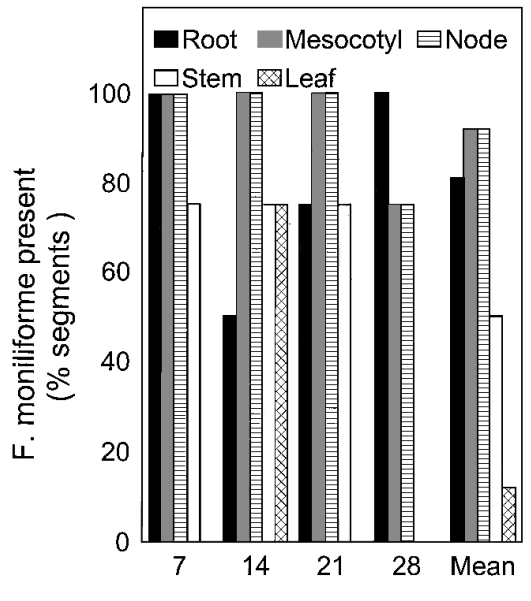

Days after planting

Fig. 2. Frequency of Fusarium moniliforme isolation during the first 4 weeks after planting from tissue segments of corn seedlings grown from inoculated kernels. The set of bars labeled mean show the means for the 4 weeks. azure II in $1 \%$ sodium borate $(1: 1, \mathrm{vol} / \mathrm{vol})$ with gentle heating of slides, and examined in a Leitz Dialux 22 EB microscope (LEICA, Wetzlar, Germany). Ultrathin sections (80 $\mathrm{nm})$ were cut, collected on 150-mesh copper grids, stained with $1 \%$ uranyl acetate in distilled water and lead citrate, and examined with a JEOL JEM-100 CS II transmission electron microscope.

\section{RESULTS}

Seed germination. Daily emerged coleoptiles did not differ significantly $(P \geq 0.05)$

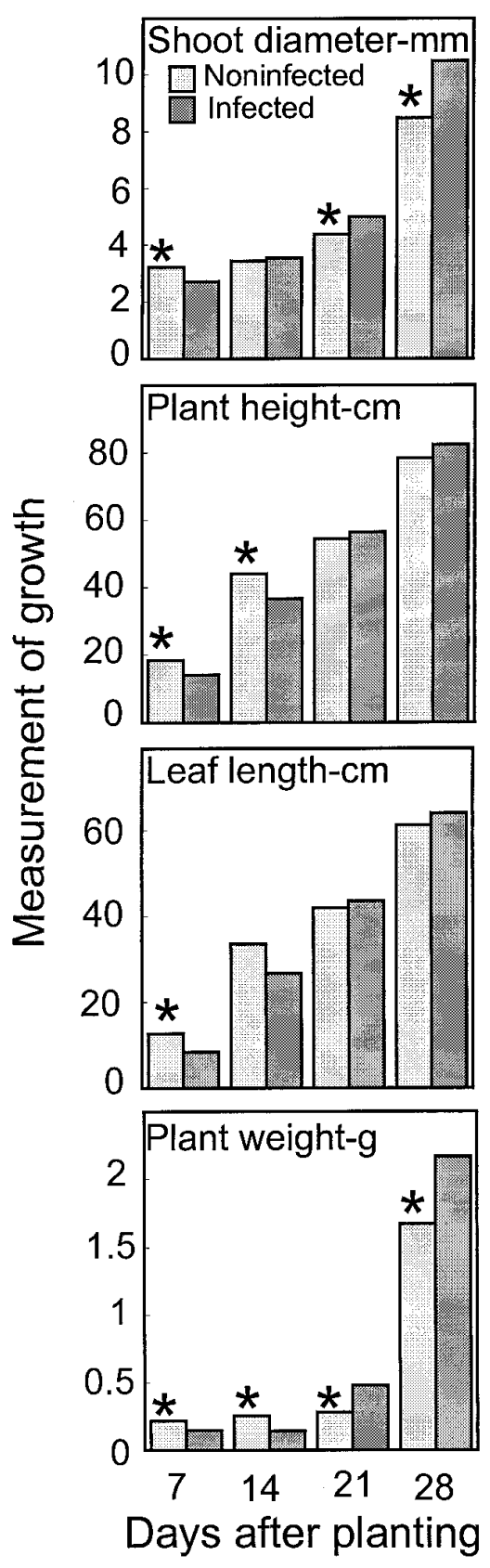

Fig. 3. Growth of corn seedlings at 7-day intervals after planting. A star above a set of bars indicates a significant difference, based on the $t$ test, between the noninfected and Fusarium moniliforme-infected plants for the indicated harvest date $(P \leq 0.05)$. 
between noninoculated and $F$. moniliforme-inoculated kernels based on data analysis by the $t$ test (Fig. 1). Nearly $80 \%$ of the kernels germinated within 3 days after planting, and cumulative coleoptile emergence followed a sigmoid-shaped curve for both noninoculated and inoculated kernels (Fig. 1). The lag phase was of short duration, because kernels were imbibed for a 24 -h period before planting. Inoculation did not affect percentages of daily and final cumulative emerged coleoptiles or onset of lag, logarithmic, and stationary phases.

$F$. moniliforme distribution in corn seedlings. F. moniliforme was never isolated from seedlings grown from noninoculated kernels in evaluations of more than 1,000 segments. In contrast, $F$. moniliforme was isolated from seedlings grown from inoculated kernels at each sampling date (Fig. 2), but the frequency in specific plant parts varied among sampling dates. The highest mean infection was found in roots, mesocotyls, and nodes, and the lowest percent infection was found in stems and leaves. Henceforth, seedlings grown from noninoculated and $F$. moniliforme-inoculated kernels will be referred to as noninfected and infected seedlings, respectively.

Seedling growth. Shoot diameter at 7 days after planting was significantly $(P=$ $0.02)$ larger for noninfected than for $F$. moniliforme-infected corn seedlings (Fig. 3). However, shoot diameter of infected seedlings equaled at day 14 and exceeded at days 21 and 28 after planting that of noninfected seedlings. Plant height at days 7 and 14 and leaf length at day 7 of noninfected seedlings exceeded that of infected seedlings but were equal at later sampling dates (Fig. 3). Dry weights of noninfected and infected seedlings were significantly different at all sampling dates, with noninfected seedlings exceeding infected seedlings at 7 and 14 days; however, infected seedlings exceeded noninfected seedlings at 21 and 28 days (Fig. 3). Disease symptoms did not appear in either noninfected or infected seedlings for the experimental duration.

Anatomical analyses of leaves, shoots, and roots. Plant cell deterioration and fungal hyphae were absent at all growth stages in leaves from noninfected and $F$. moniliforme-infected seedlings examined by LM, SEM, or TEM. Leaf anatomical features of noninfected and infected seedlings were typical of classic descriptions for the $\mathrm{C}_{4}$ photosynthetic leaves of $Z$. mays (11). However, chloroplast location and orientation, especially in bundle sheath cells, were different between noninfected and $F$. moniliforme-infected seedlings at all four weekly collections. The characteristic location for chloroplasts was adjacent to the cell wall, with the longest plastid side oriented parallel to the wall, in leaves from noninfected seedlings but was in the central portion of the cell, with the longest plastid side perpendicular to the cell wall, in leaves from infected seedlings.

Anatomical differences between shoots from noninfected and $F$. moniliforme-infected seedlings were evident at 14 days after planting. Fresh sections of shoots from noninfected corn seedlings (Fig. 4A) did not stain as intensely for lignin as shoots from $F$. moniliforme-infected seedlings (Fig. 4B). The intense staining from noninfected seedlings was apparent as a concentric band near the outer periphery of shoots. Results with fresh sections were confirmed by examination of fixed thin sections. A band of cells with thickened walls located interior to the outermost ring of vascular bundles corresponded to the region of intense staining in fresh sections. Leaves and shoots of infected seedlings had no atypically shaped or degenerated cells. Fungal hyphae were detected in fixed sections of shoots by LM, and their intercellular location was confirmed by TEM.

The integrity of root cells was maintained during the fixation process at day 7 in noninfected seedlings (Fig. 5A) but not in F. moniliforme-infected seedlings (Fig 5B). Root cell cohesion in infected seedlings was disrupted, and cellular debris was
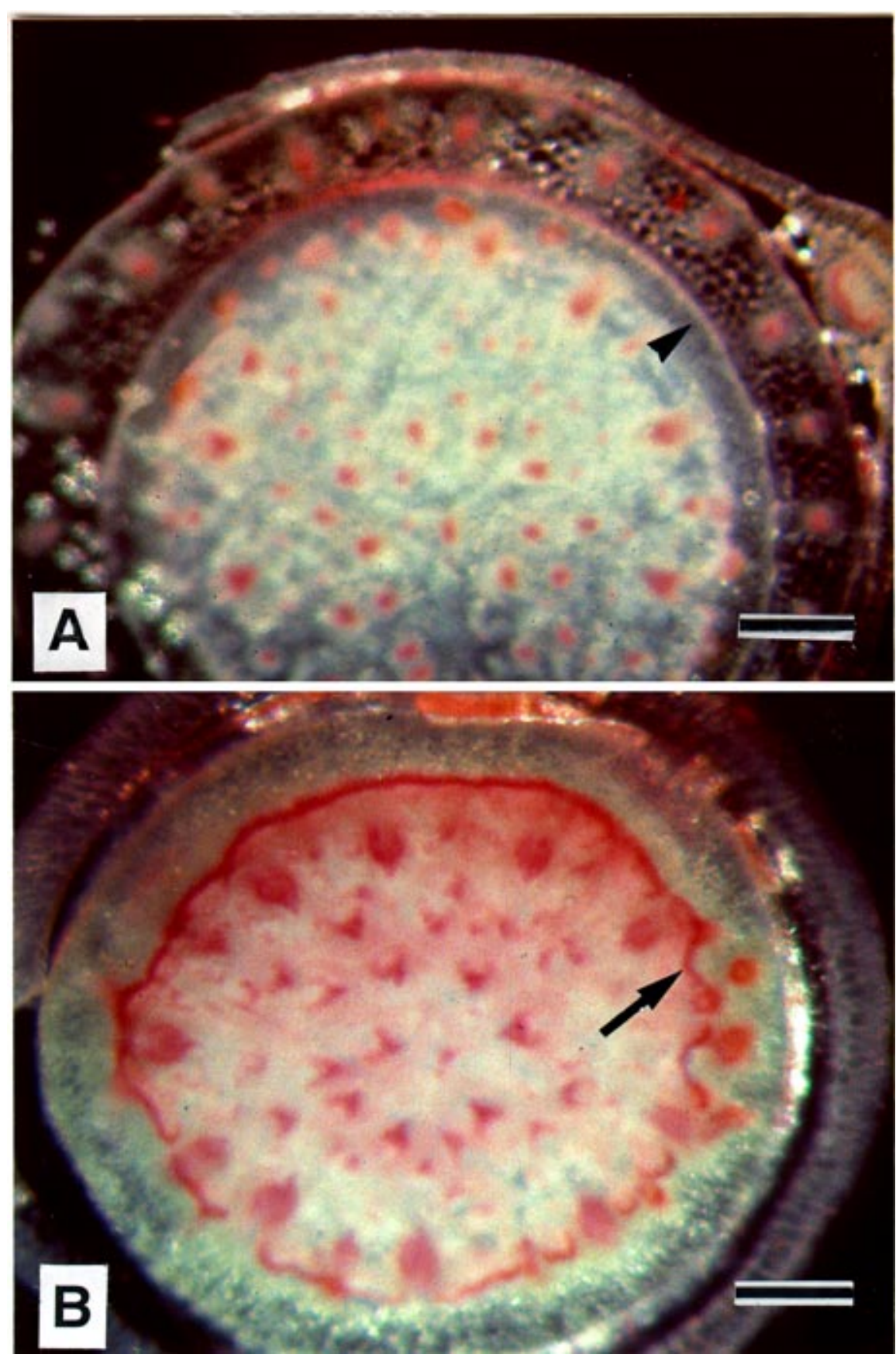

Fig. 4. Shoot sections from (A) noninfected and (B) Fusarium moniliforme-infected corn seedlings at day 14 after planting. The arrowhead (A) and arrow (B) designate the zone that differs between the two shoots for lignin staining intensity. Bars $=0.5 \mathrm{~mm}$ 

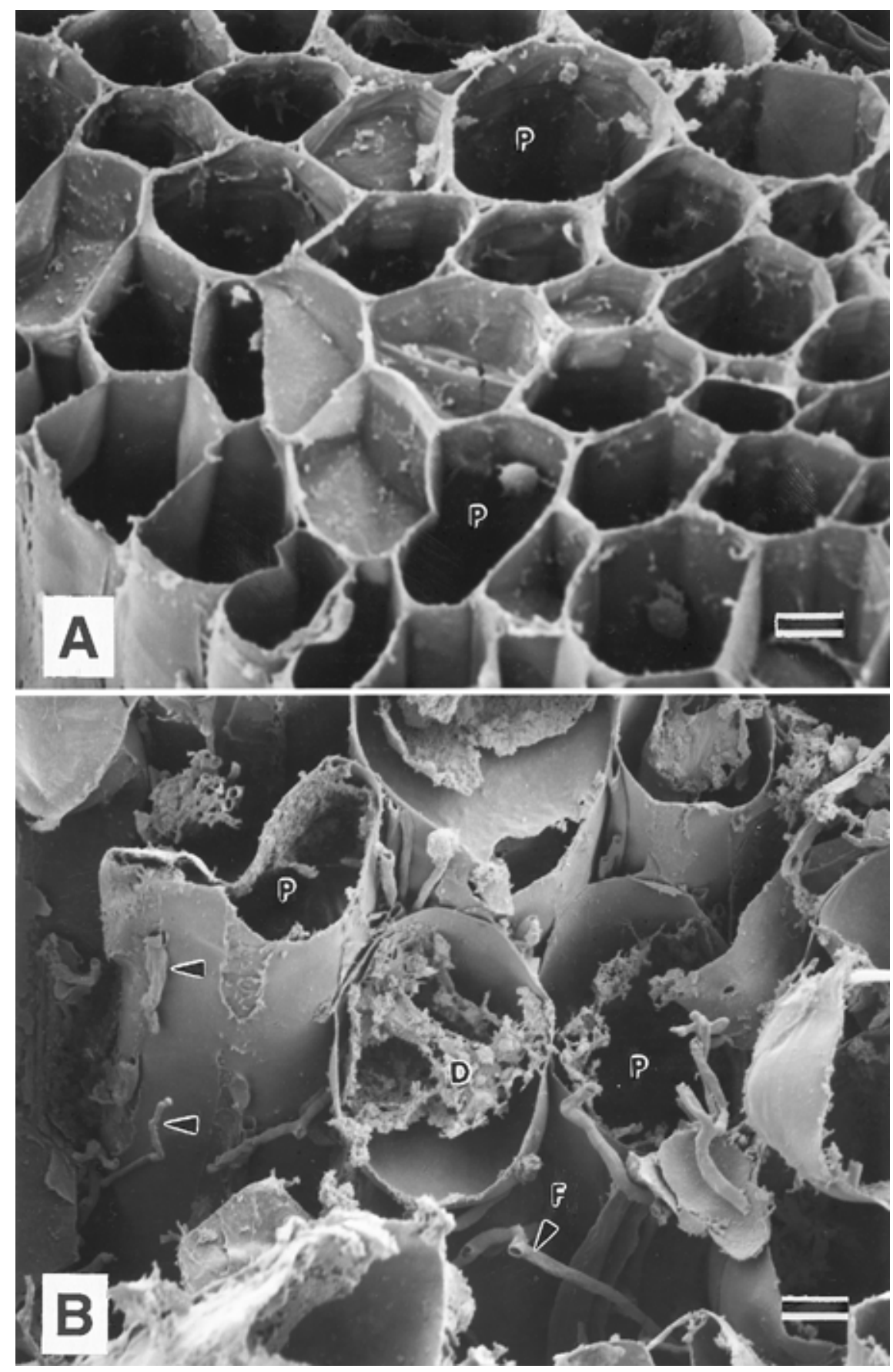

Fig. 5. Scanning electron micrographs of corn roots from (A) noninfected and (B) Fusarium moniliforme-infected corn seedlings at day 7 after planting. $\mathrm{D}=$ cytoplasmic debris; $\mathrm{F}=$ fungal hypha; and $\mathrm{P}=$ plant cell. Bars $=10 \mu \mathrm{m}$.

scattered throughout the sections. A few scattered fungal hyphae were evident at intercellular sites in roots of infected seedlings (Fig. 5B). In spite of differences in the root structure of noninfected and infected seedlings macroscopically and microscopically (Fig. 5) at day 7, root dry weight by day 28 was significantly $(P=0.04)$ higher for infected $(264.9 \mathrm{mg})$ than for noninfected $(184.3 \mathrm{mg})$ kernels. This difference was associated with shorter adventitious roots and fewer secondary roots in noninfected (Fig. 6A) compared to infected (Fig. 6B) seedlings. grasses have been the most thoroughly studied, which has led to an understanding of their benefit to plant performance through enhancement of plant vigor, pest resistance, and stress tolerance (13).

Little information exists on the dependence or interdependence of the plant and fungus in symptomless infections of corn by $F$. moniliforme. Past studies on symptomless infections of tall fescue by a fungal endophyte that produces mycotoxins harmful to cattle (6) underscore the need for analysis of the impact of symptomless infection by $F$. moniliforme on corn plants. Much time and financial resources were devoted to developing fungal endophyte-free fescue; however, the endophyte was discovered to be critical to plant growth and development $(9,26)$, making endophyte-free tall fescue agronomically useless. Another incentive for analyzing the $F$. moniliformecorn interaction is possible plant growthregulator production by the fungus (31). A major class of plant-growth hormones, gibberellin, was isolated first from the teleomorph G. fujikuroi in a classic study by Yabuta et al. (34). Furthermore, a mycotoxin of $F$. moniliforme, fumonisin $\mathrm{B}_{1}$, accelerated adventitious root formation in tomato (3). Our results demonstrated that root dry weight of $F$. moniliforme-infected corn seedlings exceeded that of roots from noninfected seedlings at 28 days after planting.

Corn kernels inoculated with $F$. moniliforme were not altered in the percentage or rate of kernel germination, but early seedling growth was influenced. Growth of $F$. moniliforme-infected, compared to noninfected, seedlings was suppressed initially. However, growth repression was temporary, because growth of infected seedlings accelerated to equal or exceed that of noninfected seedlings at 4 weeks after planting. Growth of seedlings during the first few days after germination from inoculated kernels may be limited by nutrient availability, as reported for endophyte-infected seedlings of tall fescue (9). The fungus and seedling may compete for food reserves stored in the kernel endosperm.

Intercellular hyphal growth within corn plant tissues of $F$. moniliforme strains that cause symptomless infection (2) is a characteristic of the endophytic fungal habit in symbiotic grass relationships (6). Endophytic fungi usually are confirmed by isolating the organism from surface-disinfected plant tissue, as in the current study. We isolated $F$. moniliforme from the root, mesocotyl, node, and stem of infected corn seedlings on every sampling date but from leaves at only 14 days after planting. Microscopic evidence confirmed the presence of $F$. moniliforme in roots and shoots but not in leaves. We have no definitive explanation for these results, other than that the techniques used lack the sensitivity to detect fungi with limited distribution, as was observed in other plant tissues (1). 


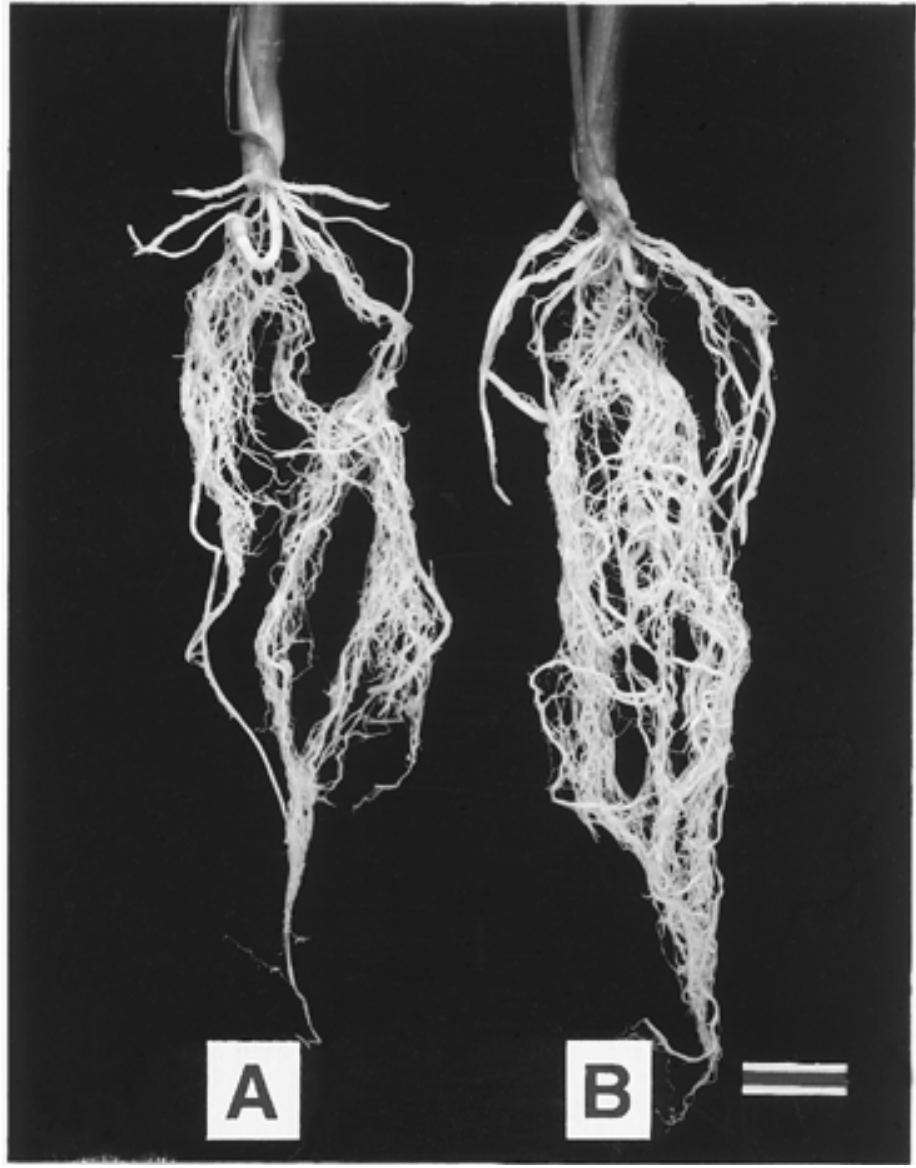

Fig. 6. Comparison of root growth of (A) noninfected and (B) Fusarium moniliforme-infected corn seedlings at day 28. Bar $=10 \mathrm{~mm}$.

Fungal pathogens invading plant hosts generally activate defense mechanisms restricting colonization. The anatomical feature of accelerated lignin deposition in shoots of $F$. moniliforme-infected corn seedlings may account for observed increases in stem rigidity and may be a structural mechanism generated by this association to hinder fungal growth into stems. Lignin can function as a mechanical barrier for organisms without a lignin-degrading enzyme $(8,17,30)$.

In summary, our study demonstrated enhanced root growth and histological modifications in leaves and shoots of corn seedlings during an endophytic, symptomless infection by $F$. moniliforme. F. moniliforme can cause severe diseases, such as blight of seedlings and rot of kernels, roots, and stalks $(18,19)$. As our studies indicate, because some endophytic strains may benefit early seedling growth, the impact of symptomless infections on crop yields of mature plants should be examined.

\section{LITERATURE CITED}

1. Bacon, C. W., Bennett, R. M., Hinton, D. M., and Voss, K. A. 1992. Scanning electron microscopy of Fusarium moniliforme within asymptomatic corn kernels and kernels associated with equine leukoencephalomalacia. Plant Dis. 76:144-148.

2. Bacon, C. W., and Hinton, D. M. 1996. Symptomless endophytic colonization of maize by Fusarium moniliforme. Can. J. Bot. 74:1195-
1202.

3. Bacon, C. W., Hinton, D. M., Chamberlain, W. J., and Norred, W. P. 1994. De novo induction of adventitious roots in excised shoots of tomatoes by fumonisin B1, a metabolite of Fusarium moniliforme. J. Plant Growth Regul. 13:53-57.

4. Bacon, C. W., Hinton, D. M., and Richardson, M. D. 1994. A corn seedling assay for resistance to Fusarium moniliforme. Plant Dis. 78: 302-305.

5. Bacon, C. W., and Nelson, P. E. 1994. Fumonisin production in corn by toxigenic strains of Fusarium moniliforme and Fusarium proliferatum. J. Food Prot. 57:514-521.

6. Bacon, C. W., and Siegel, M. R. 1988. Endophyte parasitism of tall fescue. J. Prod. Agric. 1:45-55.

7. Bacon, C. W., and Williamson, J. W. 1992. Interactions of Fusarium moniliforme, its metabolites and bacteria with corn. Mycopathologia 117:65-71.

8. Børja, I., Sharma, P., Krekling, T., and Lönneborg, A. 1995. Cytopathological response in roots of Picea abies seedlings infected with Pythium dimorphum. Phytopathology 85:495501.

9. Clay, K. 1990. Fungal endophytes of grasses. Annu. Rev. Ecol. Syst. 21:275-297.

10. Daniels, B. A. 1983. Elimination of Fusarium moniliforme from corn seed. Plant Dis. 67: 609-611.

11. Esau, K. 1977. Anatomy of Seed Plants. John Wiley \& Sons, New York.

12. Foley, D. C. 1962. Systemic infection of corn by Fusarium moniliforme. Phytopathology 52: 870-872.

13. Funk, C. R., Belanger, F. C., and Murphy, J. A. 1994. Role of endophytes in grasses used for turf and soil conservation. Pages 201-209 in: Biotechnology of Endophytic Fungi of Grasses. C. W. Bacon and J. F. White, Jr., eds. CRC Press, Boca Raton, FL.

14. Gomez, K. A., and Gomez, A. A. 1984. Statistical procedures for agricultural research. John Wiley \& Sons, New York.

15. Kedera, C. J., Leslie, J. F., and Claflin, L. E. 1994. Genetic diversity of Fusarium section Liseola (Gibberella fujikuroi) in individual maize stalks. Phytopathology 84:603-607.

16. Koehler, B. 1942. Natural mode of entrance of fungi into corn ears and some symptoms that indicate infection. J. Agric. Res. 64: 421-442.

17. Kolattukudy, P. E., Kämper, J., Kämper, U., Gonzáles-Candelás, L., and Guo, W. 1994. Fungus-induced degradation and reinforcement of defensive barriers of plants. Pages 67-79 in: Host Wall Alterations by Parasitic Fungi. O. Petrini and G. B. Ouellette, eds. The American Phytopathological Society Press, St. Paul, MN.

18. Kommedahl, T., and Windels, C. E. 1981 Root-, stalk-, and ear-infecting Fusarium species on corn in the USA. Pages 94-103 in: Fusarium: Disease, Biology, and Taxonomy. P. E. Nelson, T. A. Toussoun, and R. J. Cook, eds. The Pennsylvania State University Press, University Park.

19. Kommedahl, T., Windels, C. E., and Stucker, R. E. 1979. Occurrence of Fusarium species in roots and stalks of symptomless corn plants during the growing season. Phytopathology 69:961-966.

20. Leslie, J. F., Pearson, C. A. S., Nelson, P. E. and Toussoun, T. A. 1990. Fusarium spp. from corn, sorghum, and soybean fields in the central and eastern United States. Phytopathology 80:343-350.

21. Marasas, W. F. O., Nelson, P. E., and Tousson, T. A. 1984. Toxigenic Fusarium Species. The Pennsylvania State University Press, University Park.

22. Marasas, W. F. O., Wehner, F. C., van Rensburg, S. K., and van Schlkwyk, D. J. 1981. Mycoflora of corn produced in human esophageal cancer areas in Transkei. Phytopathology 71: 792-796.

23. Norred, W. P. 1993. Fumonisins-Mycotoxins produced by Fusarium moniliforme. J. Toxicol. Environ. Health 38:309-328.

24. Pennypacker, B. W. 1981. Anatomical changes involved in the pathogenesis of plants by Fusarium. Pages 400-408 in: Fusarium: Disease, Biology, and Taxonomy. P. E. Nelson, T. A. Toussoun, and R. J. Cook, eds. The Pennsylvania State University Press, University Park.

25. Perkins, D. D. 1962. Preservation of Neurospora stock cultures with anhydrous silica gel. Can. J. Microbiol. 8:591.

26. Rice, J. S., Pinkerton, B. W., Stringer, W. C., and Unersander, D. J. 1990. Seed production in tall fescue as affected by a fungal endophyte. Crop Sci. 30:1303-1305.

27. Schneider, H. 1981. Phloroglucinol in $\mathrm{HCl}$ for wound gum. Page 338 in: Staining Procedures. G. Clark, ed. Williams \& Wilkins, Baltimore.

28. Spurr, A. R. 1969. A low-viscosity epoxy resin embedding medium for electron microscopy J. Ultrastruct. Res. 26:31-43.

29. Stone, J. K., Viret, O., Petrini, O., and Chapela I. H. 1994. Histological studies of host penetration and colonization by endophytic fungi. Pages 115-126 in: Host Wall Alterations by Parasitic Fungi. O. Petrini and G. B. Ouellette, eds. The American Phytopathological Society Press, St. Paul, MN.

30. Vance, C. P., Kirk, T. K., and Sherwood, R. 
T. 1980. Lignification as a mechanism of disease resistance. Annu. Rev. Phytopathol. 18:259-288.

31. Voigt, K., Schleier, S., and Brückner, B. 1995. Genetic variability in Gibberella fujikuroi and some related species of the genus Fusarium based on random amplification of polymorphic DNA (RAPD). Curr. Genet. 27:528-535.

32. Warren, J. L., and Kommedahl, T. 1973. Prevalence and pathogenicity to corn of Fusarium species from corn roots, rhizosphere, residues, and soil. Phytopathology 63:1288-1290.
33. White, J. F., Jr. 1987. The widespread distribution of endophytes in the Poaceae. Plant Dis. 71:340-342.

34. Yabuta, T., Kambe, T., and Hayashi, T. 1934. Biochemistry of 'bakanae' fungus of rice. J. Agric. Chem. Soc. Jpn. 10:1059-1068. 